Case Report

Recurrent hydrothorax due to diaphragmatic perforation post hepatocellular carcinoma radiofrequency ablation:

\section{A case report}

\author{
Laia Aceituno ${ }^{1 *}$, Alba Prió ${ }^{1}$, Fernando L Ascanio ${ }^{3}$, Xavier \\ Serres-Créixams ${ }^{4}$, Beatríz Mínguez ${ }^{1,2}$, Joan Genescà ${ }^{1,2}$, \\ Lluís Castells ${ }^{1,2}$ and Ares A Villagrasa ${ }^{1}$
}

'Liver Unit, Hospital Universitari Vall d'Hebron, Vall d'Hebron Research Institute (VHIR), Universitat Autònoma de Barcelona, Vall d'Hebron Barcelona Hospital Campus, Barcelona, Spain

${ }^{2}$ Centro de Investigación Biomédica en Red de Enfermedades Hepáticas y Digestivas (CIBEREHD), Madrid, Spain

${ }^{3}$ Thoracic surgery Unit, Vall d'Hebron Barcelona Hospital Campus, Barcelona, Spain

${ }^{4}$ Radiology Unit, Vall d'Hebron Barcelona Hospital Campus, Barcelona, Spain

\section{Abstract}

Radiofrequency ablation is one of the most commonly used therapies for potentially curative small hepatocellular carcinoma. Although radiofrequency is usually a safe procedure, severe and potentially fatal complications can happen. This is a case of a 72 years old woman with cirrhosis secondary to Metabolic Associated Fatty Liver Disease (MAFLD), who presented recurrent hydrothorax after treating hepatocellular carcinoma $(\mathrm{HCC})$ with radiofrequency ablation (RFA), and in which diaphragmatic perforation was subsequently diagnosed. We reviewed the differential diagnosis and management in a pleural effusion after a radiofrequency procedure. Although diaphragmatic perforation is an uncommon complication after RFA procedure, this case aims to help clinicians being aware of non-habitual complications.

\section{More Information}

*Address for Correspondence: Laia Aceituno, Liver Unit, Hospital Universitari Vall d'Hebron, Vall d'Hebron Research Institute (VHIR), Universitat Autònoma de Barcelona, Vall d'Hebron Barcelona Hospital Campus, Barcelona, Spain,

Tel: 0034637942905 ;

Email: laceituno@vhebron.net;

laiaaceituno@gmail.com

Submitted: February 01, 2021

Approved: February 13, 2021

Published: February 15, 2021

How to cite this article: Aceituno $L$, Prió $A$, Ascanio FL, Serres-Créixams X, Mínguez B, et al. Recurrent hydrothorax due to diaphragmatic perforation post hepatocellular carcinoma radiofrequency ablation: A case report. Ann Clin Gastroenterol Hepatol. 2021; 5: 013-015.

DOI: 10.29328/journal.acgh.1001026

ORCiD: orcid.org/0000-0001-5414-2408

Copyright: @ 2021 Aceituno L, et al. This is an open access article distributed under the Creative Commons Attribution License which permits unrestricted use, distribution, and reproduction in any medium, provided the original work is properly cited.

Keywords: Cirrhosis; Pleural effusion Radiofrequency ablation; Diaphragmatic perforation; Hepatocellular carcinoma

Check for updates

O open Access

\section{Background}

Hepatocellular carcinoma (HCC) is an aggressive tumor that often occurs in the setting of chronic liver disease and cirrhosis and therapeutic options are determined both by tumoral stage and the severity of the underlying liver disease.

Treatment options are divided into potentially curative therapies (hepatic resection, orthotopic liver transplantation or percutaneous ablation including among other techniques: radiofrequency ablation, cryoablation, microwave) and palliative therapies (i.e. transarterial chemoembolization, or systemic therapy) [2].

Among ablative therapies, one of the most commonly used is radiofrequency ablation (RFA) which is considered a potentially curative therapeutic option and commonly accepted as the best alternative for patients with small HCCs $(<3 \mathrm{~cm}$ in diameter) who are not candidates for surgical resection or liver transplantation [2]. Although RFA is a minimally invasive procedure, well tolerated and with a low percentage of side effects, severe and potentially fatal complications can occur.

Here, we report an uncommon complication of RFA presented as a recurrent hydrothorax 6 months after the procedure due to a diaphragmatic perforation.

\section{Case report}

A 72-year-old woman was admitted because of recurrent hydrothorax (Figure 1). The patient had liver cirrhosis secondary to Metabolic Associated Fatty Liver Disease (MAFLD) in Child-Pugh's stage B (8 points). The patient had previously presented an esophageal variceal bleeding. During follow-up, a $27 \mathrm{~mm}$ HCC was diagnosed in segment VI (Figure 2), that was successfully treated by RFA in May 2018. The patient was followed in the outpatient clinic 
without evidence of tumor recurrence until 18 months later, when a new $15 \mathrm{~mm} \mathrm{HCC}$ was detected by CT scan in segment VI/VII (Figure 3), hydrothorax was not present at that time. A new RFA was performed in September 2019 without complications. Six months after the procedure, the patient was admitted to the hospital for dyspnea. A right hepatic hydrothorax was observed, and a thoracentesis confirmed the presence of a pleural effusion compatible with a transudate (protein $1.9 \mathrm{~g} / \mathrm{dL}$, LDH $110 \mathrm{IU} / \mathrm{L}$ ) without signs of infection and no malignant cells in the cytological extension. Despite repeated thoracocentesis and diuretic treatment, pleural effusion recurred. A thoraco-abdominal CT showed a cirrhotic liver without new lesions, moderate ascites, splenomegaly, patent portal vein with a splenorenal shunt, and moderate pleural effusion without pleural or lung lesions.

With the intention of performing a chemical pleurodesis, a video-assisted thoracoscopy surgery (VATS) was performed demonstrating the presence of a diaphragmatic perforation $4 \mathrm{~cm}$ in diameter (Figure 4 ) which was surgically repaired. In the immediate postoperative period, a pleural drainage was required due to hydrothorax recurrence. After 10 weeks, the patient remains without pleural fluid despite having abdominal ascites that has required multiple evacuating paracentesis.

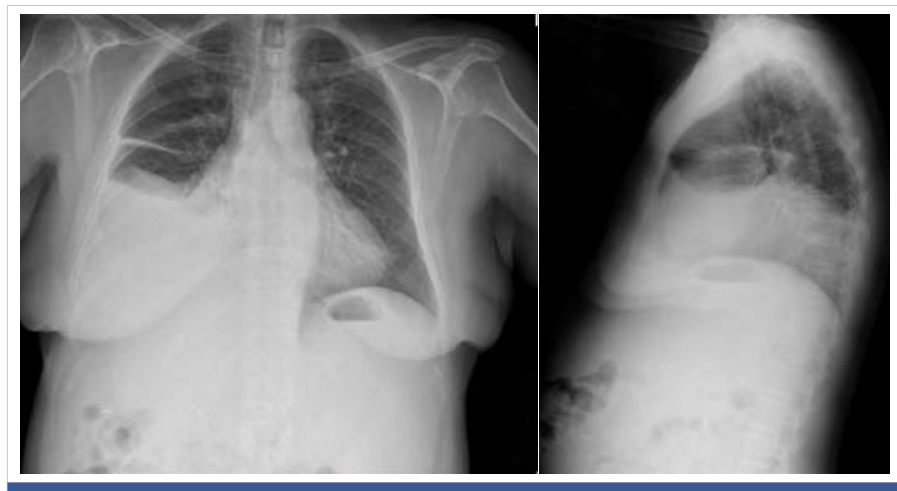

Figure 1: Anteroposterior and lateral chest X-ray showing right pleural effusion.

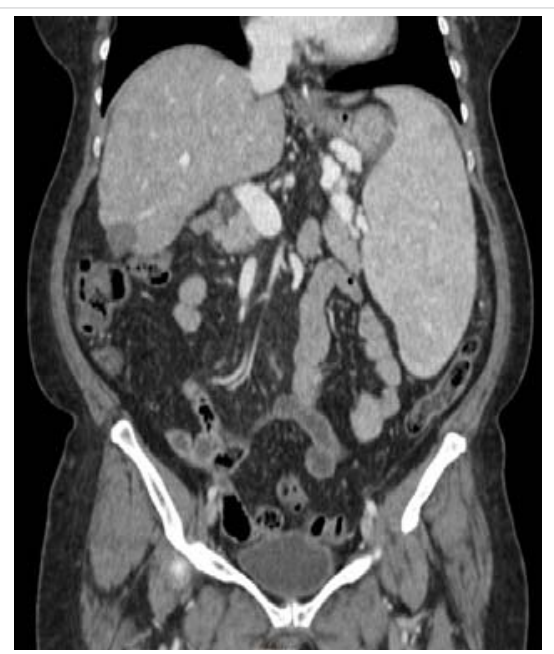

Figure 2: Abdomen CT scan. Hypodense residual post RFA lesion of $27 \mathrm{~m}$ in segment VI.

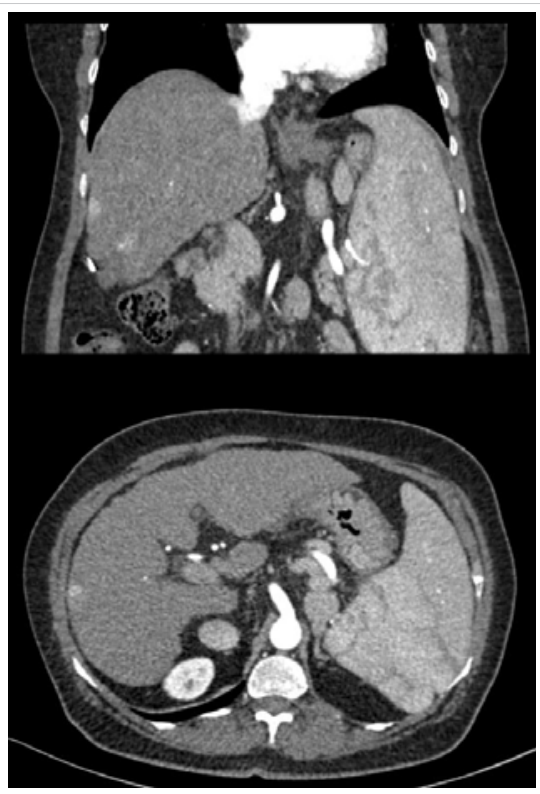

Figure 3: Abdomen CT scan. Subcapsular hypervascular nodules in segment VIIVII.

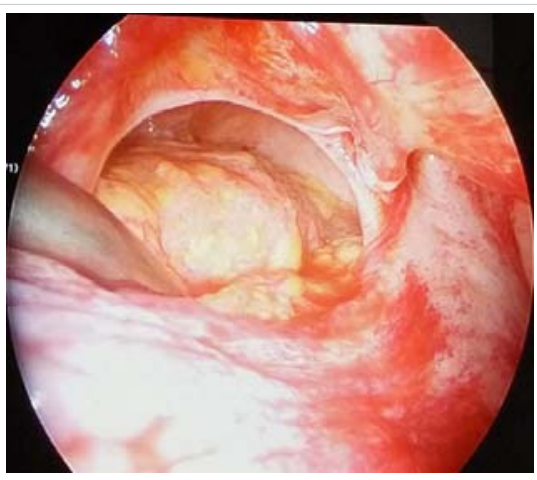

Figure 4: Video-assisted thoracoscopy surgery (VATS). Diaphragmatic perforation of $4 \mathrm{~cm}$ in diameter.

\section{Discussion}

RFA provides long-term local control for most selected patients with small hepatocellular carcinoma, with the possibility of long-term disease-free survival. Complete radiographic response rates (complete necrosis) following RFA are $90 \%$ to $99 \%$ for tumors $<5 \mathrm{~cm}$, with the highest and most consistent response rates for lesions $\leq 3 \mathrm{~cm}[5,6]$.

Although RFA is relatively well tolerated, severe and potentially fatal complications can occur. Major complications develop in $2 \%$ to $11 \%$ of procedures, and the mortality rate is $0.1 \%$ to $0.8 \%[4,5]$. Among the most frequent complications, it is worth to mention haemorrhagic complications (intraabdominal or intra-hepatic bleeding, subcapsular hematoma or haemobilia), liver abscess, skin burns, pneumothorax or pleural effusion. Diaphragm perforation (with or without herniation) is a rare major complication [7]. The mechanism of diaphragm perforation after RFA has not been clarified yet, although three causative factors have been described: tumor location, collateral thermal injury and advanced cirrhosis status. Similar to our case, location is one of the most important causative factors. This is especially 
important in peripheral subcapsular tumors, since the risk of diaphragmatic thermal injury increases, as the visualization by the radiologist using ultrasounds is complicated due to the lung and ribs interference. Although not as well studied, the incidence of pleural effusion is about $0.1 \%$, with a similar rate for diaphragmatic hernias [7].

Hepatic hydrothorax is the presence of pleural effusion in a patient with cirrhosis without evidence of cardiopulmonary disease. It occurs in 5\% - 15\% of patients with cirrhotic portal hypertension and is associated with high mortality [1].

It has been proposed that the combination of positive intraabdominal pressure and negative intrathoracic pressure facilitates the movement of fluid from the peritoneal cavity into the pleural space through defects in the diaphragm.

The differential diagnosis of hepatic hydrothorax in HCC patients who undergo RFA includes malignant, infectious and diaphragm injury-related pleural fluid. The study of the pleural effusion after RFA should include a diagnostic thoracentesis with biochemistry, cytological extension and microbiological culture. In addition, a thoracic CT scan is mandatory to rule out the presence of a lung or pleural tumor, or any anatomical defects. Hepatic hydrothorax can occasionally be diagnosed through direct visualization of diaphragmatic defects by VATS, although this procedure is invasive and should be performed only if the diagnosis remains unclear or it is planned to repair the defect.

There are some techniques to prevent or minimize diaphragmatic thermal injury mostly related to tumor location issues, such as artificial ascites, artificial pleural effusion, carbon dioxide insufflation or laparoscopy assisted ablation [2].

Approximately 50 RFA are performed per year since 2004 in our hospital. This is the first documented case of hydrothorax secondary to a diaphragmatic defect caused by this procedure in our Liver Unit. Another case was detected years ago in our center, but we couldn't find enough data to report it.

In conclusion, the present report presents a case of recurrent hepatic hydrothorax due to a diaphragmatic perforation after RFA. Although this complication is uncommon, clinicians should be aware of its occurrence, and keep in mind this possibility as part of the differential diagnosis in a patient with liver cirrhosis and HCC, after a RFA procedure especially in subcapsular and subphrenic locations.

\section{References}

1. Banini BA, Alwatari $Y$, Stovall $M$, Ogden $N$, Gershman $E$, et al. Multidisciplinary Management of Hepatic Hydrothorax in 2020: An Evidence-Based Review and Guidance. Hepatology. 2020; 72: 1851-1863. PubMed: https://pubmed.ncbi.nlm.nih.gov/32585037/

2. Galle PR, Forner A, Llovet JM. EASL Clinical Practice Guidelines: Managementofhepatocellularcarcinoma. JHepatol.2018;69:182-236. PubMed: https://pubmed.ncbi.nlm.nih.gov/29628281/

3. De Baère $T$, Risse $O$, Kuoch V, Dromain $C$, Sengel $C$, et al. Adverse events during radiofrequency treatment of 582 hepatic tumors. Am J Roentgenol. 2003; 181: 695-700.

PubMed: https://pubmed.ncbi.nlm.nih.gov/12933462/

4. Livraghi T, Solbiati L, Meloni MF, Gazelle GS, Halpern EF, et al. Treatment of focal liver tumors with percutaneous radio-frequency ablation: Complications encountered in a multicenter study. Radiology. 2003; 226: 441-451.

PubMed: https://pubmed.ncbi.nlm.nih.gov/12563138/

5. Brunello F, Cantamessa A, Gaia S, Carucci P, Rolle E, et al. Radiofrequency ablation: technical and clinical long-term outcomes for single hepatocellular carcinoma up to $30 \mathrm{~mm}$. Eur J Gastroenterol Hepatol. 2013; 25: 842-849.

PubMed: https://pubmed.ncbi.nlm.nih.gov/23442417/

6. N'Kontchou G, Mahamoudi A, Aout M, Ganne-Carrié N, Grando V, et al. Radiofrequency ablation of hepatocellular carcinoma: Long-term results and prognostic factors in 235 western patients with cirrhosis. Hepatology. 2009; 50: 1475-1483.

PubMed: https://pubmed.ncbi.nlm.nih.gov/19731239/

7. Takaki H, Yamakado K, Nakatsuka A, Yamada T, Shiraki K, et al. Frequency of and risk factors for complications after liver radiofrequency ablation under ct fluoroscopic guidance in 1500 sessions: Singlecenter experience. Am J Roentgenol. 2013; 200: 658-664. PubMed: https://pubmed.ncbi.nlm.nih.gov/23436859/

8. Tamano M, Hashimoto T, Kojima K, Maeda C, Hiraishi H. Diagnosis of hepatic hydrothorax using contrast-enhanced ultrasonography with intraperitoneal injection of Sonazoid. J Gastroenterol Hepatol. 2010; 25: 383-386

PubMed: https://pubmed.ncbi.nlm.nih.gov/19817961/

9. Kim JS, Kim HS, Myung DS, Lee GH, Park KJ, et al. A case of diaphragmatic hernia induced by radiofrequency ablation for hepatocellular carcinoma. Korean J Gastroenterol. 2013; 62: 174-178. PubMed: https://pubmed.ncbi.nlm.nih.gov/24077629/

10. Lin MW, Lee JM. Video-assisted Thoracoscopic Surgery for Diaphragmatic Defect Complication With Refractory Hydrothorax Related to Radiofrequency Ablation. J Formos Med Assoc. 2010; 109: 673-675.

PubMed: https://pubmed.ncbi.nIm.nih.gov/20863995/ 